\title{
Light Duty Tracking and Sensing System Using the Adafruit web*
}

\author{
Sistema de seguimiento y sensado de carga liviana utilizando la plataforma web de Adafruit
}

Received: January 14, 2019 | Accepted: September 10, 2019 | Published: February 24, 2020

\section{Héctor Torres-Bustos}

Universidad Tecnológica Metropolitana del Estado de Chile, Chile.

ORCID: 0000-0002-4163-606X

Macarena Valenzuela-Zubiaur

Universidad Tecnológica Metropolitana del Estado de Chile, Chile.

ORCID: 0000-0003-3199-8355

\section{Víctor Meza-Herrera ${ }^{a}$}

Universidad Tecnológica Metropolitana del Estado de Chile, Chile.

ORCID: 0000-0001-9629-4880

\section{Francis Soto-Pacheco}

Universidad Tecnológica Metropolitana del Estado de Chile, Chile.

ORCID: 0000-0003-0029-5913

\section{Víctor Escobar-Jeria}

Universidad Tecnológica Metropolitana del Estado de Chile, Chile.

ORCID: 0000-0003-2455-8175

${ }^{*}$ Review article

${ }^{a}$ Corresponding author. Email: v.mezah@utem.cl

DOI: https://doi.org/10.11144/Javeriana.iyu24.ldts

\section{How to cite this article:}

H. Torres-Bustos, M. Valenzuela-Zubiaur, V. Meza-Herrera, F. Soto-Pacheco, and V. Escobar-Jeria, "Light duty tracking and sensing system using the Adafruit web," Ing. Univ., vol. 24, 2020. https://doi.org/10.11144/Javeriana.iyu24.Idts 


\section{Abstract}

A prototype developed in ProteinLab UTEM for a tracking system and parametric control for light transport loads that require cold chain fulfillment validation in the interdisciplinary work between engineering and design is presented in this article. The work is performed using fast prototyping development boards, such as Arduino MEGA, a communication shield FONA808, and sensors associated with variables such as temperature and humidity, and the recorded data are sent to the IO.Adafruit.com platform in the cloud. The information is displayed in a dashboard and stored for later export and analysis. The use of these development boards optimizes the incorporation of compatible sensors that allow for a registry of variables that are relevant for future system requirement modifications, such as weight or impact sensors. Finally, the prototype development contemplates the concept of the final product being prepared for testing with end-users as part of the working methodology of ProteinLab UTEM, Center for Technological Innovation, which explores the different methods for comprehensively addressing current technological challenges.

Keywords: Arduino, adafruit IO, cloud, FONA808, GPS, GPRS, Internet of the things, light-duty vehicles, sensors

\section{Resumen}

El artículo presenta un prototipo para un sistema de seguimiento y control paramétrico en cargas de transporte ligero que requieren validación del cumplimiento de la cadena de frío, en un ejercicio de trabajo interdisciplinario entre ingeniería y diseño desarrollado en ProteinLab UTEM. El trabajo se realiza utilizando placas de desarrollo de prototipos rápidos, como Arduino MEGA, en comunicación una placa FONA808 y sensores asociados con variables como temperatura y humedad, y los datos grabados se envían a la plataforma IO.Adafruit.com en la nube. La información se muestra en un tablero y se almacena para su posterior exportación y análisis. El uso de estas placas de desarrollo optimiza la incorporación de sensores compatibles que permiten un registro de variables que resulten relevantes para futuras modificaciones en los requisitos del sistema, como sensores de peso o impacto. Finalmente, el prototipo contempla en su diseño el concepto de producto final preparado para ser testeado con usuarios finales como parte de la metodología de trabajo de ProteinLab UTEM, Centro de Innovación Tecnológica, que explora diferentes maneras de abordar los desafíos tecnológicos actuales.

Palabras clave: Arduino, IO.Adafruit, nube, FONA808, GPS, GPRS, Internet de las cosas, vehículos menores, sensores 


\section{Abbreviations}

1-Wire A protocol communication designed by Dallas Semiconductor Corp.

$2 \mathrm{G}$

$3 \mathrm{D}$

Second-generation cellular technology

$3 \mathrm{G}$

Three-dimensional space

$4 \mathrm{G}$

Third-generation mobile cellular system

A-GPS

Fourth-generation mobile cellular system

API

APN

Assisted global positioning system

AWS

Application programming interface

GPRS

Access point name

GPS

Amazon web services

GPS L1 C/A

General packet radio service

\section{GSM}

Global Positioning System

HTTP

GPS (L1 - carrier 1575.42 MHz) coarse/acquisition

IoT

Global system for mobile communications

Hypertext transfer protocol

$\mathrm{I} / \mathrm{O}$

Internet of Things

LTE

Input or output

MQTT

Long-term evolution

OBD-II

Message queue telemetry transport

PLA

On-board diagnostics (OBD), $2^{\text {nd }}$-generation.

RH Relative-humidity

SEREMI Secretaría Regional Ministerial

SIM Subscriber identity module

SSL Secure sockets layer

TCP/IP Transmission control protocol (TCP) and the internet protocol (IP)

TLS Transport layer security

TTFF Time to first fix

UMTS Universal mobile telecommunications system 


\section{Introduction}

The present work shows the design and development of a system for tracking and sensing environmental parameters for a cargo compartment coupled to a light electric transport vehicle.

The purpose of the project is to develop a product-oriented to the contexts of innovation and entrepreneurship, which require rapid prototyping of their ideas. The efforts of this project focus on the search for a reliable solution. These attempts must be compatible with restrictions imposed either by the current health regulations of a country related to the transfer of specific products or by considerations inherent to the transport of light loads, as stated in the project development item when the objectives of the project are addressed by the prototype presentation.

The value of this project lies in introducing concepts of digital transformation such as the Internet of Things, cloud processing, and energy autonomy, into the entrepreneurship ecosystem and enabling entrepreneurs to address new requirements imposed by the current technological context through the development of hardware-based solutions.

The chosen context is the logistics of small and medium-scale fleets in merchandise transport that require follow-ups in their cold chain with goods such as food and medical supplies, for which the positioning of the fleet and the monitoring of thermal parameters or the state of the charge is of great importance. The exploration of tracking solutions is also considered, based on the development of fast implementation prototypes, which has allowed it to become a feasible solution for safeguarding the assets of a company or activity, and it is fundamental when planning logistics as it has been resolved in works [1]-[3] where they covered the use of electronic development boards as a base element of their study proposals.

The existing measures related to the field of industrial vehicular safety focus on accessories for vehicles that have installed computers and use proprietary protocols, such as OBD-II; therefore, these solutions cannot be applied in the context of this project.

\section{Project development}

The project objective is to obtain location, humidity, and temperature data during the track of a load employing a test module (prototype) that provides the user with a clear reading of the information. The main characteristics of the developed prototype are its quick implementation, low cost, and use of open-source development tools available on the Arduino and Adafruit digital platforms. 


\section{Project objectives}

The requirements of the project are summarized as follows:

- Fast implementation, where the "added value" is given by the startup of the idea more than the immediate development of new and exclusive hardware.

- Encapsulated portable electronic module for service evaluation tests.

- Lightweight.

- Low cost in electronics, computer solutions, and encapsulation manufacturing.

- Composed of location, humidity, and temperature sensors.

- Sends data to a remote server through the Internet.

- Eight-hour autonomy.

The aim of measuring the humidity and temperature is to comply with legal restrictions on the transport of food and medical supplies [4], [5] and thus, consider a broad spectrum of transport loads. In the case of Chile, the restrictions affecting the prototype border the minimum temperature spectrum at $-18^{\circ} \mathrm{C}$ for the most extensive transport time (the desired cooling) to a maximum of $-12^{\circ} \mathrm{C}$ for a short period of time (as a condition that should not be exceeded) for the transport of refrigerated meals. In the case of non-refrigerated food, the maximum temperature cannot exceed the recommendation given by the manufacturer. In the case of transportation of medical supplies, with particular emphasis on the transfer of vaccines, the temperatures to be monitored are critical and cannot exceed the range of $+2^{\circ} \mathrm{C}$ to $+8^{\circ} \mathrm{C}$, according to what is reported by the SEREMI of Health of Chile. Therefore, the emphasis on temperature management and continuous monitoring is a significant restriction in regard to projecting a solution.

\section{Development and methodology}

To comply with the stated objective, the communication module used in this work was the FONA808 development board, which is an interface board that communicates with the SIM808 chipset and has extensive literature focused on IoT solutions, all developed by the manufacturer, Adafruit $®$. In addition, employing its API application, it is possible to obtain GPS positioning parameters and connect to the cellular network via the GPRS protocol; through a microcontroller, it communicates with the internal modem that the development board has implemented. Once the information of the integrated monitoring system is obtained, this information is processed through the Adafruit IO platform to obtain the behavior of the device associated with its geolocation and the parameters that are being recorded.

Once the above is proven, the device is used in local transmission tests, where physical aspects that are linked to the transmitter module compartment and its functionality to parameterize the logistics of tracking a test load are tested. For this purpose, different traceability tests are executed with varying types of materials in the encapsulation of the device, which are detailed in the experimental section under the precept of continuous improvement methodology. 


\section{Literary review}

This process of monitoring and positioning devices can be found in various prototyping applications using the SIM908 module [1], [6], [7], which allows obtaining the positioning data and cellular communication over the GPRS/GSM network through a single integrated communication module. The reason that it was not considered for implementation of the prototype was its cost regarding the necessary services for the project.

The proposal presented in the case applied by [8] was also analyzed through the GTIM900$\mathrm{B}$ module as a communication platform with the cellular network with the U-Blox NEO-6 M module, which provides satellite positioning data, but the reason it was discarded as a solution was the low configuration documentation available for the first module.

Another case was analyzed by [9], which presented the development of an IoT framework to obtain positioning data through the communication protocol used by SIGFOX, suggesting an alternative wireless communication network of low transfer rate and long-range to communicate with devices that register satellite positioning and communicate without relying on a data SIM. However, given that the SIGFOX network is not yet deployed in Chile, it had to be discarded since the initial requirement is that it must be implemented in a short time.

Thus, the SIM808 chipset was chosen. The SIM808 chipset is a communication module that allows communication with the $2 \mathrm{G}$ cellular network (GSM/GPRS) and contains an integrated GPS modem [10] and that, in contrast to the solutions mentioned above, has extensive technical literature, and its acquisition cost is low. However, as a result of the slow and gradual withdrawal of the $2 \mathrm{G}$ cellular network in some countries, it has a diminishing life horizon, which is a significant disadvantage. The global association GSMA projects that $2 \mathrm{G}$ will have a $5 \%$ device connection rate in 2025, a situation that by the year 2018 was $35 \%$ [11]. Even so, by 2018, the infrastructure implementation of the LTE networks was still a smaller percentage than that for the $2 \mathrm{G} / 3 \mathrm{G}$ technologies.

An example of the use of this chipset can be found in works [12]-[14]. It should be noted that the authors of [12] used the module in a fleet control and used a controller that communicates with the vehicle's computer through its protocol based on OBD-II. The authors of [13] used the FONA808 module as a platform for transmitting and receiving data to a cloud platform, but with tracking functionality for people through image capture, and concatenating the visual register with the geolocation of the individual. Finally, the authors of [14] used the same communication module as an interface for their cloud positioning and traceability service through the use of MQTT as a data transmission/reception protocol.

From the information presented, we can validate that the added value in rapid prototyping for development that has restrictions in the speed of implementation time, low implementation cost and high availability in the market is solved with the technological proposal that we present in this work. The use of the SIM808 chipset responds to the necessity of proving an idea in its concept of a minimum viable product. 


\section{Light-duty vehicles and location}

The current trend is the use of smaller urban electric charging vehicles (known as "electromobility"), which are more friendly to the environment and have better mobility within a city [15]. A light-duty vehicle is one that can transport a weight that does not exceed $3,856 \mathrm{~kg}(8500 \mathrm{lb}$.) according to the US EIA Institute of Statistics and Independent Analysis, either motorized or not, and they are usually used to deliver small volumes.

It is usual for delivery services to have a tracking system [3], [6], [16], which allows knowing, at all times, the location of the goods and even tracking them online. Generally, this system is a web application, which may be owned by the company or by an external service provider, which, by handing a device to the carrier, can regularly send geolocation data and other relevant information to the logistics operation chain. However, these solutions are aimed at large company fleets, so a small entrepreneur can hardly afford their use, which reinforces the need to have a solution available quickly on a small scale.

Among the satellite location systems available on the market, the oldest and most used is GPS; considering that the prototype needs to be used in urban environments, it is necessary to have better accuracy of the mobile user's location. For this, the A-GPS system is required in densely populated environments, such as capital cities.

The geolocation of fleets in Chile is an aspect that is growing as well as the introduction of electromobility in logistics systems for industry and retail such as cargo trucks, transport for commerce stores, Correos de Chile (see Figure 1), bus companies (Transantiago and urban buses), among others. These systems at the industry level have advanced geolocalization and digital logistics systems that allow them, through sensors (the type of sensor depends on the project and the manufacturer), to obtain information on the cargo they transport, their positioning, the desired destination and other parameters that may be relevant for the logistics operations of a company.

Figure 1. Correos de Chile's electric vehicle

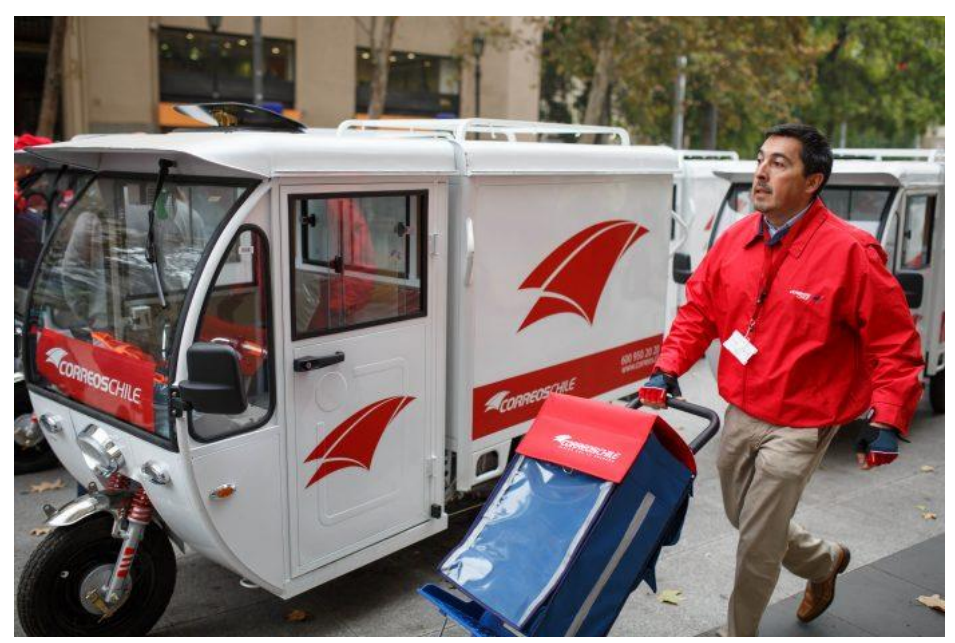

Source: La Nacion News Journal. 
Although the implementation of light-duty electric vehicles has increased due to international companies, small businesses show a greater interest in their use as an effective means of transport in densely populated environments given the advantage of size, cost, and mobilization, in terms of what represents an electric vehicle over a combustion vehicle [17][19] in the same conditions.

\section{Presentation of the prototype}

There are two types of development boards associated with prototyping that are commonly used: those that have a microcontroller, such as Arduino and its variations, and others that use a microprocessor, such as Raspberry Pi and BeagleBone. A significant difference in the complexity of the subsequent design of an embedded solution exists; a microprocessor requires a higher number of components and must have an operating system for its use, which is why they have a higher cost, and a microcontroller requires essential elements that are easily acquired for its operation.

Additionally, microcontrollers only need the associated programming for the tasks it has to perform, without requiring a full operating system, so its low cost of implementation is desirable. From the range of solutions presented by Arduino, the Arduino MEGA board was chosen, which has a higher number of connection pins, both analog and digital, and a suitable programming memory for the use of libraries related to sensors and communication modules. Since the Arduino MEGA board is based on the ATmega2560 Atmel microcontroller, unlike the most common boards such as Arduino UNO, NANO or Pro mini, which are based on the integrated ATmega328p, and due to its memory and number of pins, does not allow use with a larger quantity or complexity of sensors.

In the case of choosing a communication protocol, there are multiple eligible technologies to choose from for the means of communication, but the cellular network has the highest percentage of penetration, giving better operational options in both urban and rural environments. Among the available technologies are GSM, usually referred to as $2 \mathrm{G}$, UMTS commonly known as $3 \mathrm{G}$, and LTE or $4 \mathrm{G}$; finally, the oldest $2 \mathrm{G}$ coverage was selected since it has the most extensive coverage network available from all telephone operators that have cellular service in Chile, regardless of whether it is a city or a purely rural environment. While the $2 \mathrm{G}$ service is being phased out, and its shutdown is progressive worldwide, the chipsets that allow the use of the network are more economical and do not hinder a future transition to another technology, either $3 \mathrm{G}, 4 \mathrm{G}$ or even the use of wide-area networks and low power, such as Sigfox, LoRa, NB-IoT, when available.

With the selected communication protocol, the different options compatible with Arduino were evaluated to avoid increasing the difficulty of implementation and subsequent development of an embedded system. Because of this, the FONA808 board from Adafruit was chosen, which has extensive literature that supports its use and three hardware options: modular type, shield type, and independent socket. For this work, we chose the dockable version with an Arduino UNO board. 
The main features of this module are as follows [20]:

- Connection to the GSM network with a quad-band: 850/900/1800/1900 MHz frequency range.

- Sending and receiving text messages.

- Data transfer (TCP / IP, HTTP) via general packet radio system.

- AT command interface.

MT3337 GPS integrated circuit:

- Twenty-two tracking channels and 66 acquisition channels.

- GPS code L1 C/A.

- Tracking sensitivity $-165 \mathrm{dBm}$; cold sensitivity $-147 \mathrm{dBm}$.

- Time to first fix:

○ Cold start: 32 seconds, typical.

- Warm start: 5 seconds, typical.

○ Hot start: 1 second, typical.

- Approximate accuracy: 2.5 meters.

Although FONA808 is not the least expensive option at the market level, the technical documentation and the constant updates of libraries (repository of software) given by the manufacturer of the Adafruit module, together with the clear examples of its use besides the IO, Adafruit sites allow us to expedite the presentation of a solution of easy understanding and with possibilities for expansion.

For the initial tests, the AM2302 sensor was chosen, which measures ambient temperature and humidity. Although it is not accurate and durable in the range considered acceptable for industrial-level technological developments, it complies with the requirements and with our objective to be low cost and easy to acquire. Its technical characteristics are listed below:

- 1-wire digital signal for transmission protocol.

- Operating ranges:

○ Humidity: $0 \%$ to $100 \%$ relative humidity $(\mathrm{RH})$.

○ Temperature: $-40^{\circ} \mathrm{C}$ to $80^{\circ} \mathrm{C}$.

- Precision:

○ Humidity: $\pm 2 \%$ RH, $\pm 5 \%$ RH maximum.

$\circ$ Temperature $< \pm 0.5^{\circ} \mathrm{C}$.

- Resolution:

O Humidity: $0.1 \%$ RH.

○ Temperature: $0.1^{\circ} \mathrm{C}$.

Once the sensor was defined, programming the sensor was performed through the use of libraries provided by the manufacturer, Adafruit, to achieve reliable readings of ambient temperature and humidity. This programming was left in operation for an extended period to 
verify the values obtained followed those indicated by meteorology services; satisfactory results were obtained.

Upon solving the options, both means for the reception and sending of the positioning information of the vehicle and the variables of temperature and environmental humidity (either by satellite triangulation or by cellular communication networks [1]) that are managed by the device must be transmitted to a service that allows storing, processing and visualizing the collected data. There are two commonly used solutions for this: a local server or cloud storage [8], [21], [22].

- Local server: the storage of information on a local server, as shown in the diagram in Figure 2, requires a high investment in infrastructure and hardware necessary for processing large quantities of data that can arrive simultaneously. This option involves personnel specialized both in the maintenance of the equipment and in the computer science associated with the analysis of information.

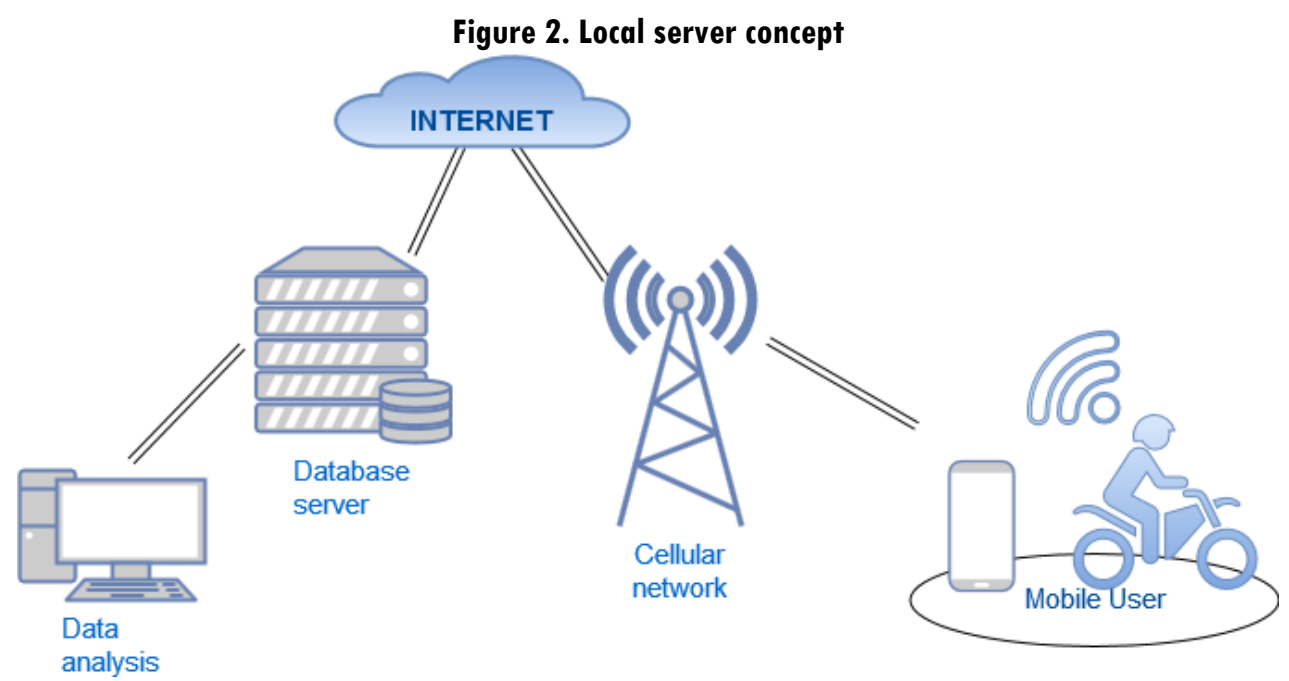

Source: Authors' own creation.

- Server in the cloud: storage in the cloud generates significant savings of time and personnel associated with the maintenance of the necessary infrastructure in local servers since these become services accessed via the Internet, as shown by the scheme in Figure 3. These services have, in most cases, both data storage service and tools that allow an exhaustive analysis, with which it is possible to generate continuous process and procedure improvement of the companies. The most commonly used services are Amazon Web Service, Microsoft Azure, IBM Bluemix, and the Google Cloud Platform. 
Figure 3. Server in the cloud concept

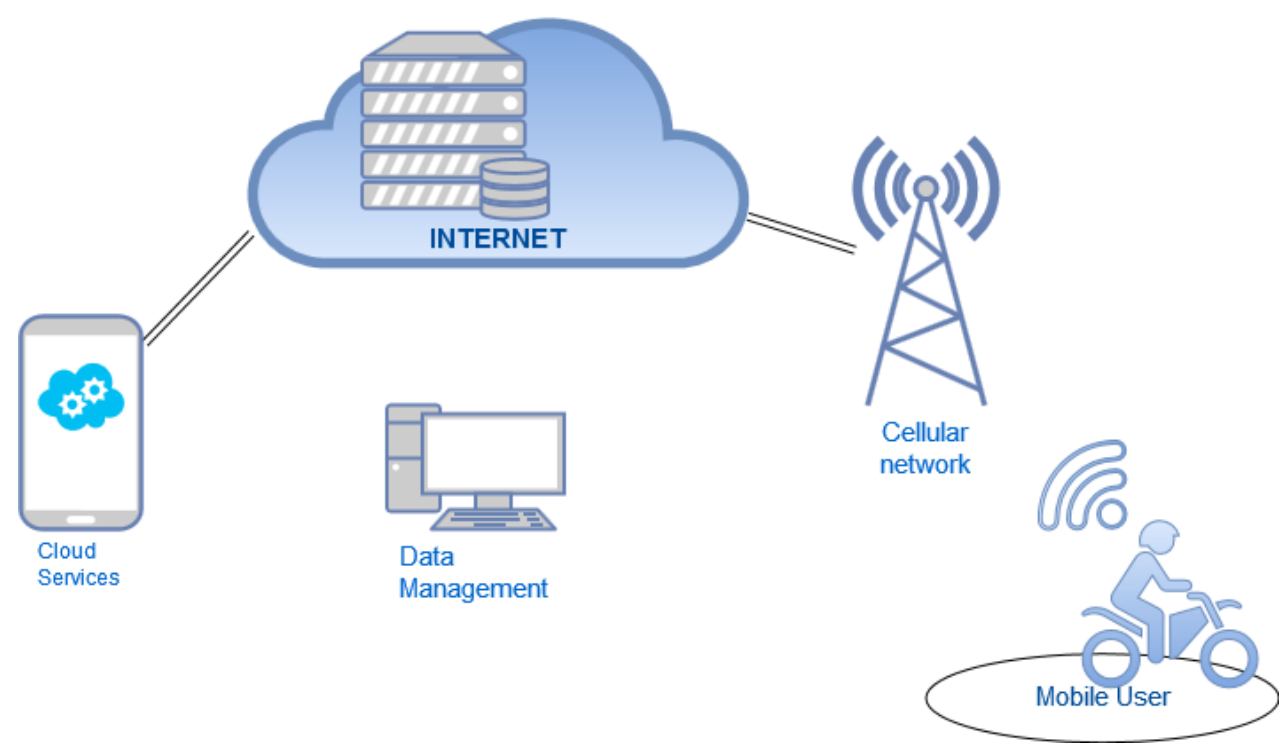

Source: Authors' own creation.

Other storage options in the cloud only consider the possibility of storing and, in some cases, visualizing the data sent by the module such as the Carriots platform, SensorCloud, and ThingSpeak. There are services with free options for the performance of low impact tests, which in most cases are limited to one or two devices and no more than ten variables. These services are commonly associated with the solutions implemented for the so-called Internet of Things (IoT).

For these tests, it was decided to opt for the website provided by the Adafruit IO. Adafruit, rather than larger platforms, was chosen mainly because they require advanced computing knowledge, and for extensive use of the modules, payment must be made. The Adafruit IO allowed us, in its free version, to save the data for one month and has different display options that include real-time graphics and incorporates maps that show the geolocation information, allowing us to track the displacement of the module, in addition to having clear literature for any user.

The programming stage of the FONA808 communication module was subdivided into two actions:

- Programming for the GPS position tests: The programming test for the communications module was loaded to verify that it could precisely and reliably identify the location through the GPS network. After the execution of adjustments to improve the accuracy of the locating, this stage was considered satisfactory.

- Programming for data transmission tests via GPRS / GSM: The test programming of the data transmission was loaded, and it was also necessary to create a user to review the information transmitted to the website provided by the manufacturer, io.adafruit.com; the test succeeded in sending simple data such as the state of charge of the battery used. 
- When satisfactory results were obtained with both programming, all the programs were combined to send the data of interest, such as the environmental temperature, humidity, GPS coordinates, speed of GPS module, and loading status of the battery. The communication procedure is shown in the following algorithm in Figure 4:

Figure 4. Programming flowchart

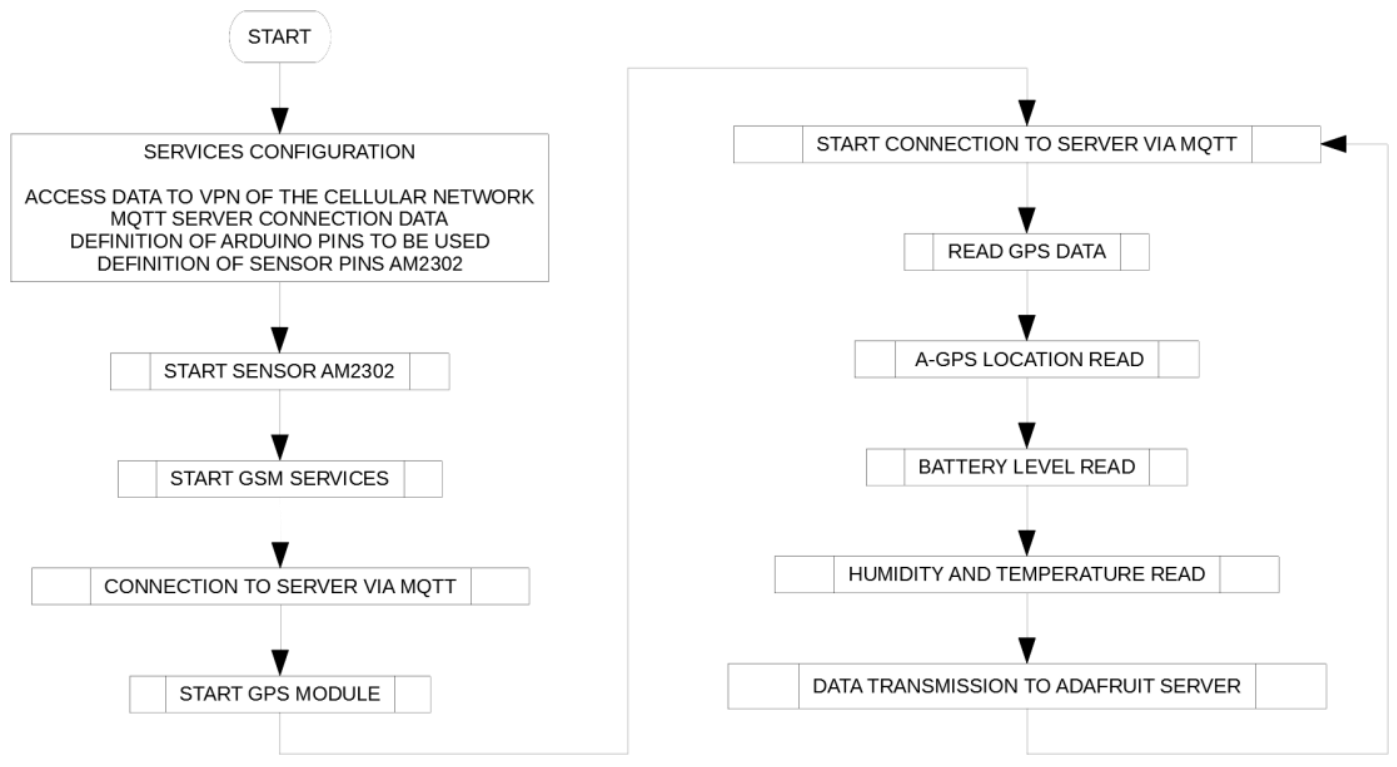

Source: Authors' own creation.

In the algorithm described above, we can identify the initialization of the AM2302 sensor; then, it starts the negotiation procedure with the GSM network through the use of the data entered in the APN section. Once this stage is successful, the GPS module initializes. To begin the synchronization, at this point, the main loop that reads the AM2302 sensor is initiated and requests the location information from the GPS module to send the data to the test website of the manufacturer Adafruit through the use of the MQTT protocol, which proceeds with the data entered in the server section.

The possibility of using asymmetric communication for transmission of less weight, considering the future incorporation of more devices makes the MQTT protocol the best alternative for sending the data to the server [23]. The described cycle is repeated until the module is switched off. Figure 5 shows an example of this information. 
Figure 5. Received data in the Adafruit 10 server

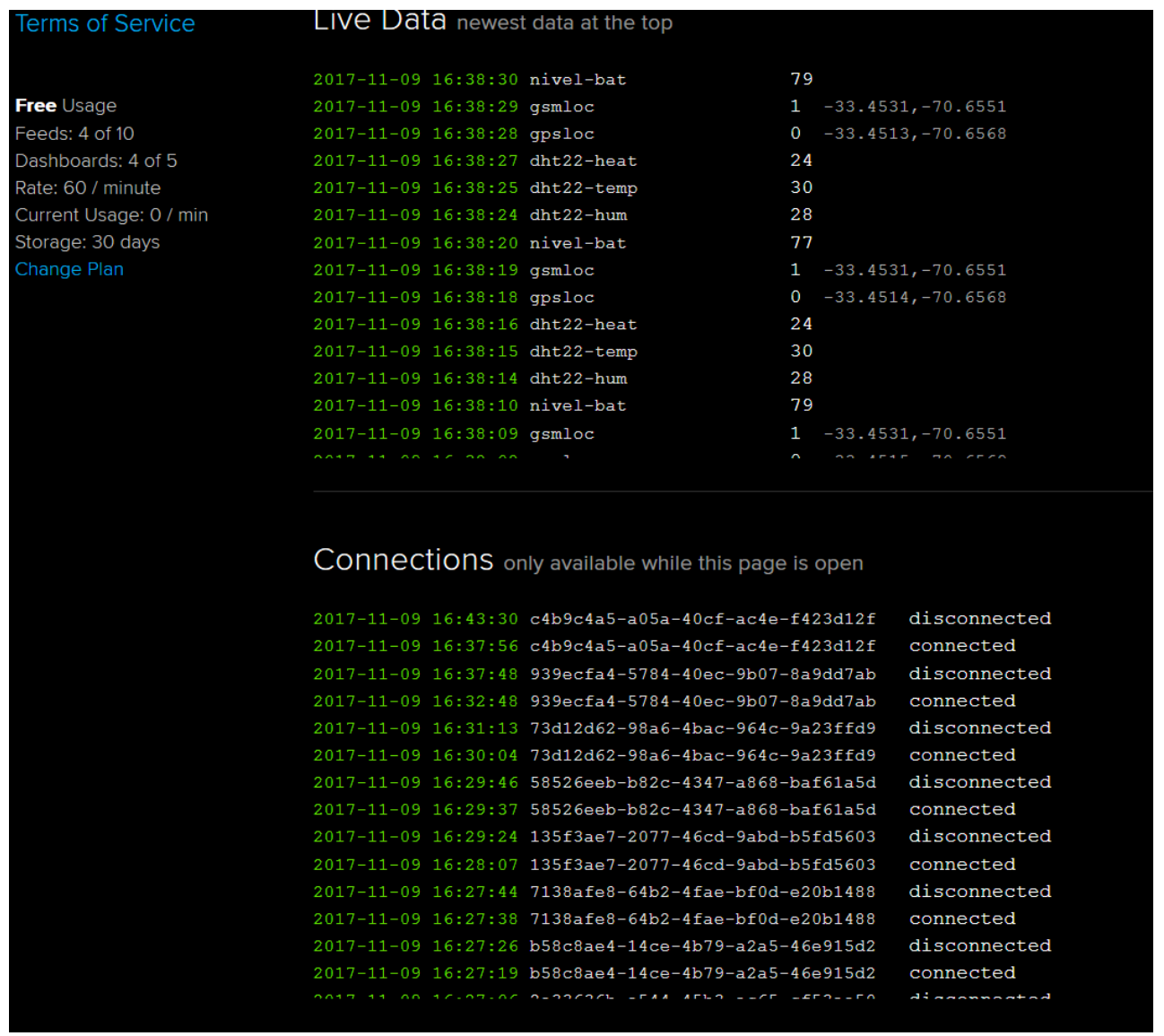

Source: Authors' own creation.

\section{Experiments}

Prototyping work was performed where the emphasis was placed on satellite positioning and communication through the cellular system to perform real-time position identification, in addition to the parameters sensed inside the smaller electric vehicle. This stage of experimentation was divided into three parts:

- First Stage: Three initial tests were carried out as proof of concept. The purpose of this was to evaluate the problems and possible improvements to be implemented, focusing on the transmission of information.

- Second Stage: Once the problems detected were solved and the improvements implemented, we carried out performance tests under controlled environments in which the prototype could be found in light-duty transportation.

- Third Stage: This last stage concentrated the final modifications and generated a final prototype for tests, considering encapsulation according to the possible environments in which the device was found. 
As seen from the description of these three stages, the baseline methodology used corresponds to the continuous improvement cycle, emphasizing the checkpoint where proper analysis of the results of the tests allowed us to make significant improvements, thereby requiring a smaller number of iterations to achieve the objectives.

\section{First test stage}

Initial test

The initial test consisted of verifying the performance of the module in an urban environment, evaluating the quality of the link with the cellular network, the accuracy of the location according to the GPS network, the response of the humidity and temperature sensor, and the response of the service in the cloud. For this, an encapsulation model made of cardboard was used to avoid any communication problem due to the use of a material that does not allow good reception of the cellular signal and GPS.

A pedestrian route of 10 to 15 minutes was followed; during this time, the module's response was continuously monitored through the Adafruit IO website, focusing on the connection status and GPS location data.

The results of this test in terms of location show that there was a problem sending the position before the GPS module synchronized (Cold Start); therefore, the accuracy was not adequate. All data were received and displayed without problems in the Adafruit IO, as shown in Figure 6.

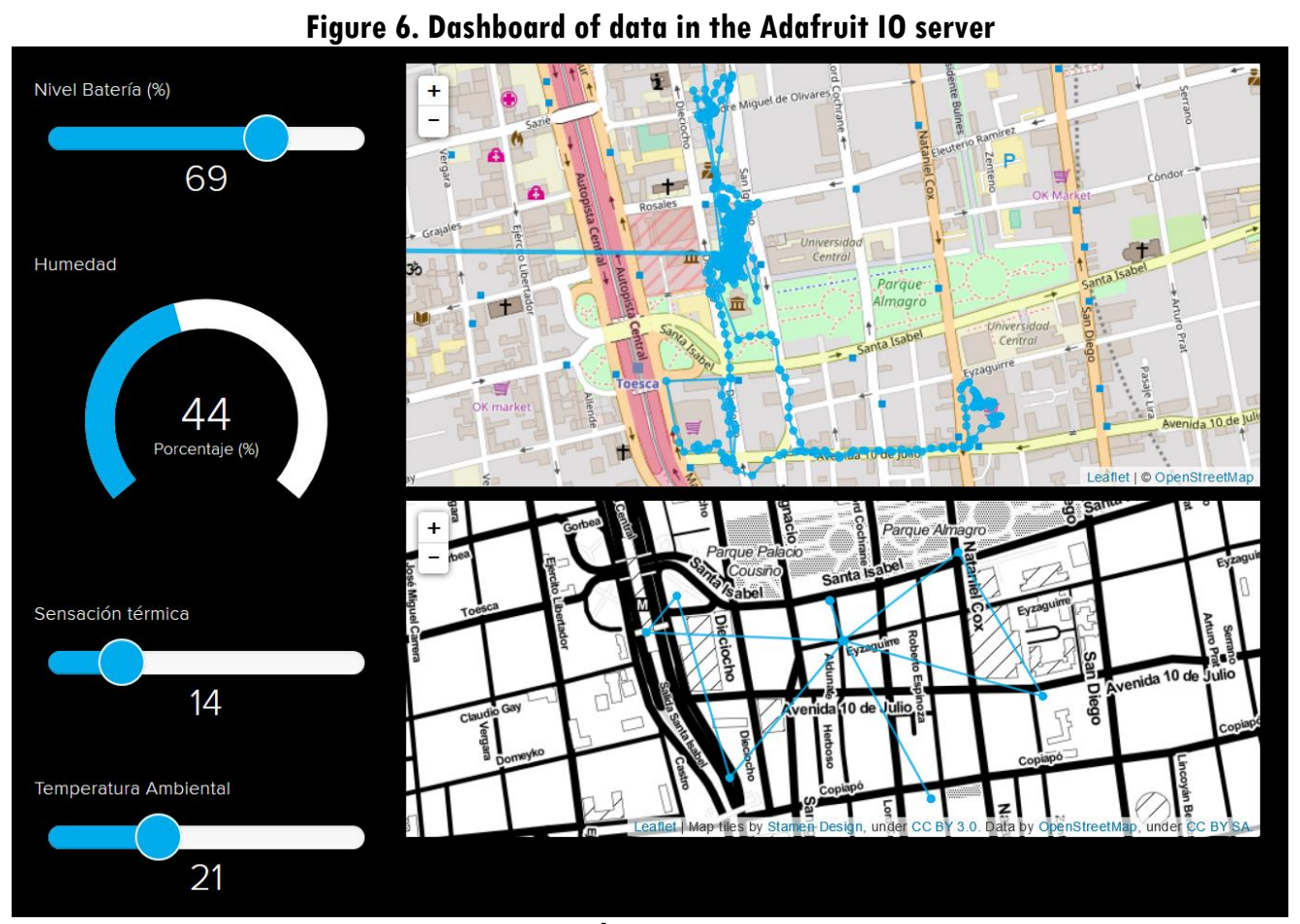

Source: Authors' own creation. 


\section{Second test with improvements}

The second test was carried out with an encapsulation made by PLA using the 3D printing method, whereby the module had no communication problems and was of compact dimensions. With this version, a more extensive route was made to capture the correct number of location points and obtain a significant quantity of data. This route was followed by a pedestrian and lasted between 20 and 30 minutes and was repeated three times. The connection and disconnection rate was minimal, which was expected for a communication system via the cellular network, and a large quantity of data was obtained, which is shown in Figure 7.

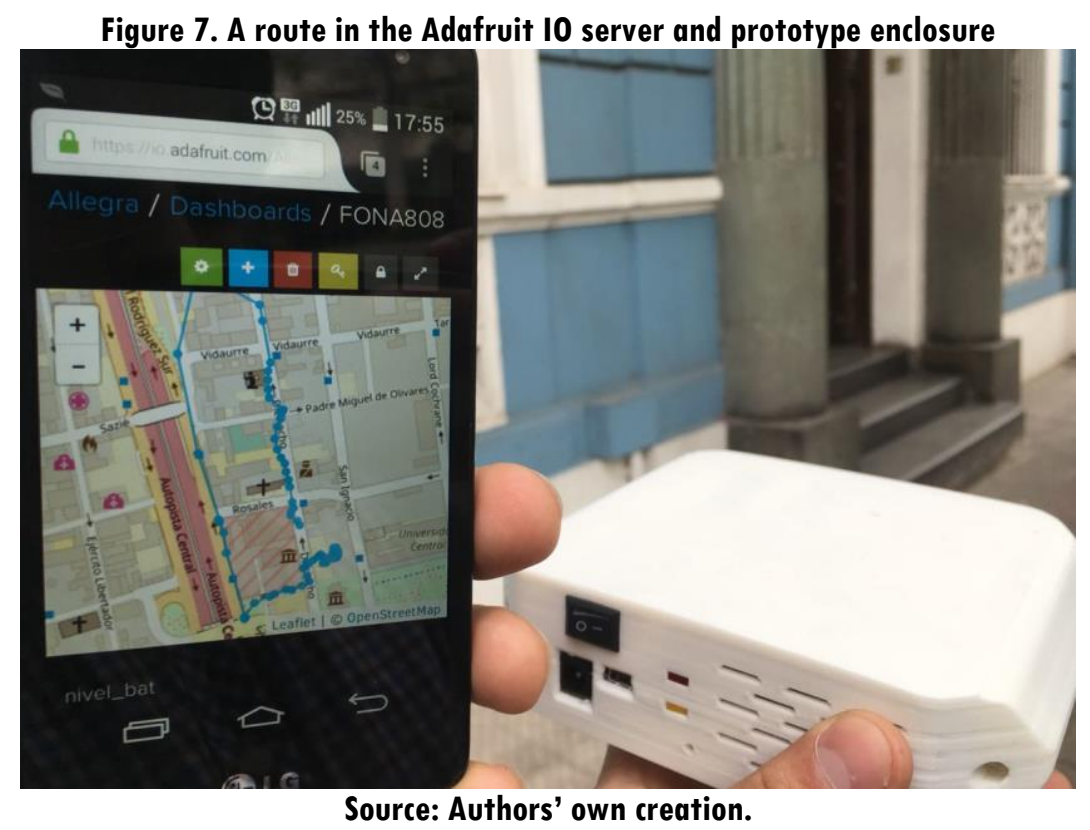

\section{Test driver}

The third and last test was performed inside a car keeping the PLA encapsulation and visualizing the differences between the GPS points obtained with a pedestrian movement and one with the speed of displacement of a vehicle in the city. With this, it was observed that the module responded correctly in terms of latitude and longitude data submitted through the GPS. Even when having several incidents of connection and disconnection, these were not significant for the large quantity of data received and did not interfere in mobile tracking. The route is seen in Figure 8. 
Figure 8. Card route in the Adafruit 10 server

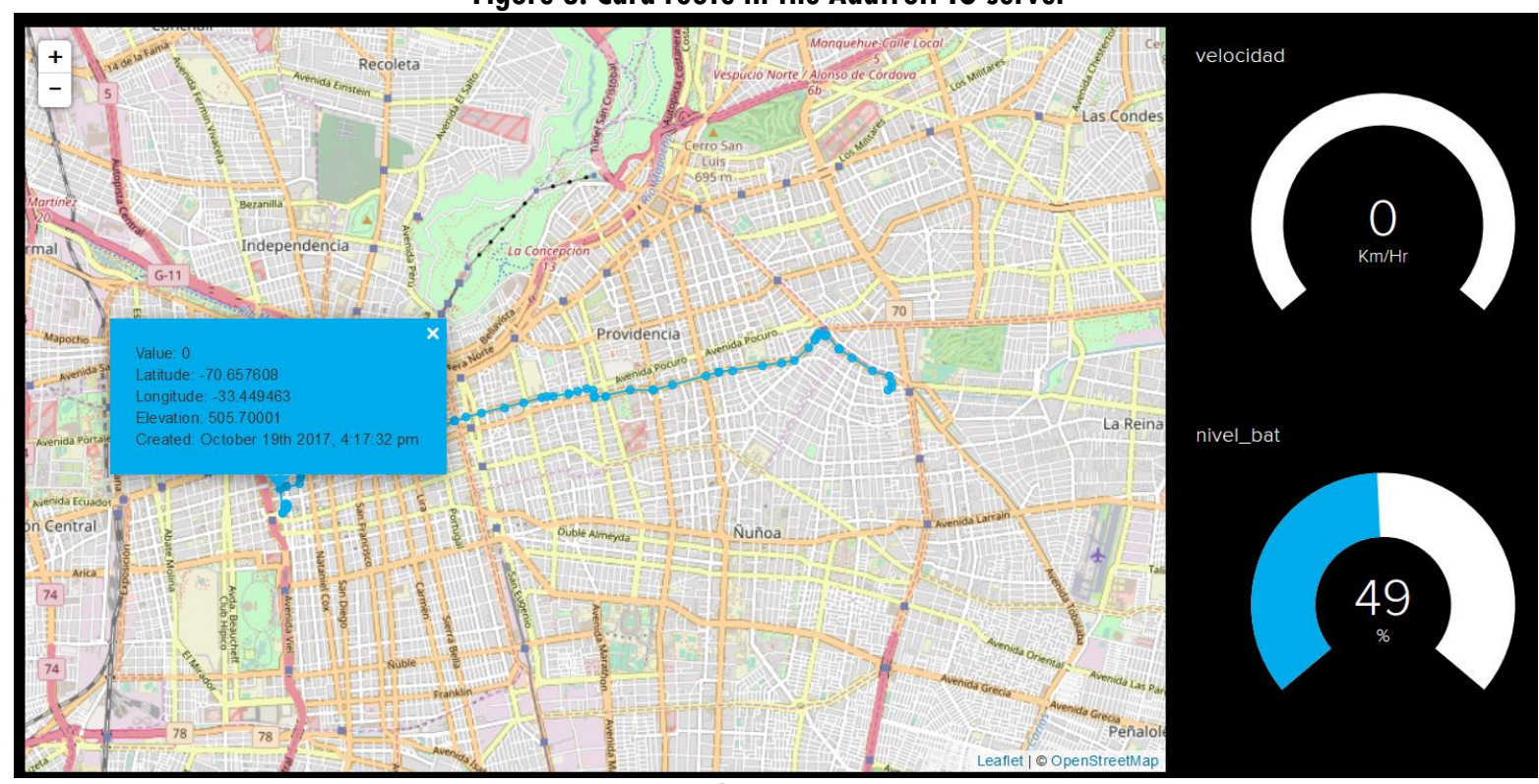

Source: Authors' own creation.

\section{The second stage of tests}

Before starting this stage, we made improvements in terms of programming the module to optimize both the quantity of data sent and the battery life since the constant sending harms the lifetime that the module can be maintained working. However, this is optional if it considers that the relevant data are useful for improving the disponibility of the device tracker.

Therefore, it was concluded for our proposal devices that the best options for sending the location data were as follows:

- The initial latitude and longitude parameters were verified, and if these were outside the Chilean territory, they were not sent to the server.

- The location data was only sent if the vehicle was moving at speeds higher than 10 $\mathrm{kph}$.

- In the case of speed lower than $10 \mathrm{kph}$, it was determined whether the vehicle moved forward more than 2 meters.

- Any other case was considered as the vehicle not in motion, and therefore, the location was not sent until there was displacement.

In the case of the other variables of interest, the following criteria were considered:

- Battery level; only if there was a variation of $1 \%$ of the load level.

- Environmental temperature; only if there was a variation of $1^{\circ} \mathrm{C}$.

- Thermal sensation; only if there was a variation of $1{ }^{\circ} \mathrm{C}$.

- Humidity; only if there was a variation of $1 \% \mathrm{RH}$. 
- Speed; only if there was a variation of $1 \mathrm{kph}$.

With this, a variability verification step was added to the algorithm. By doing so, we ensured a reasonable minimum for tests related to sending data to the server, and therefore, a saving of the transmission costs was obtained, as well as better energy performance. The final operating algorithm is shown in Figure 9.

\section{Figure 9. Corrected programming flowchart}

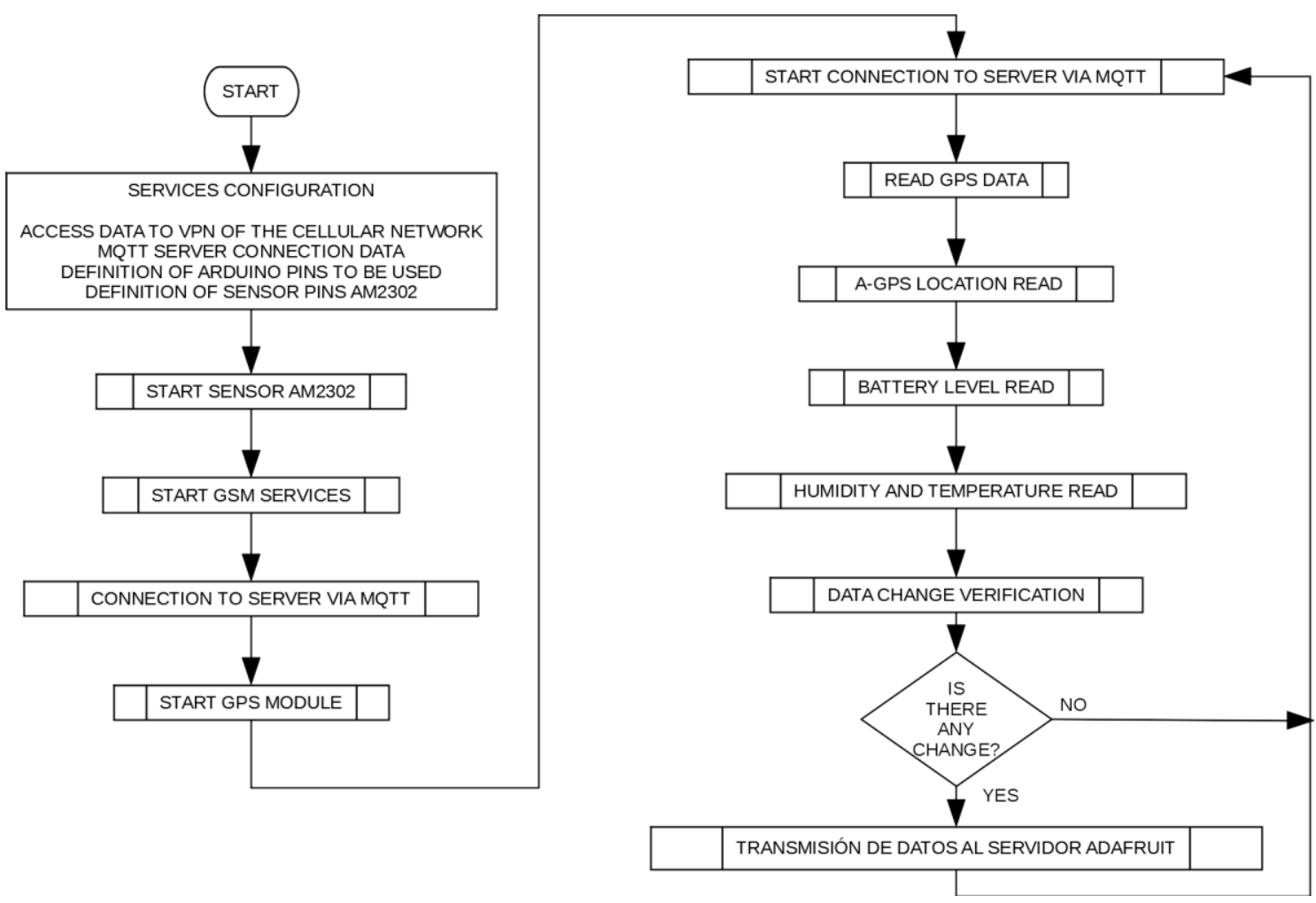

Source: Authors' own creation.

Once the programming details were solved, the pedestrian path tests were repeated considering the duration of the lithium polymer battery used, which had a capacity of 1200 $\mathrm{mAH}$ for the analysis on that occasion (see Figure 10). 
Figure 10. Battery lifetime of the prototype

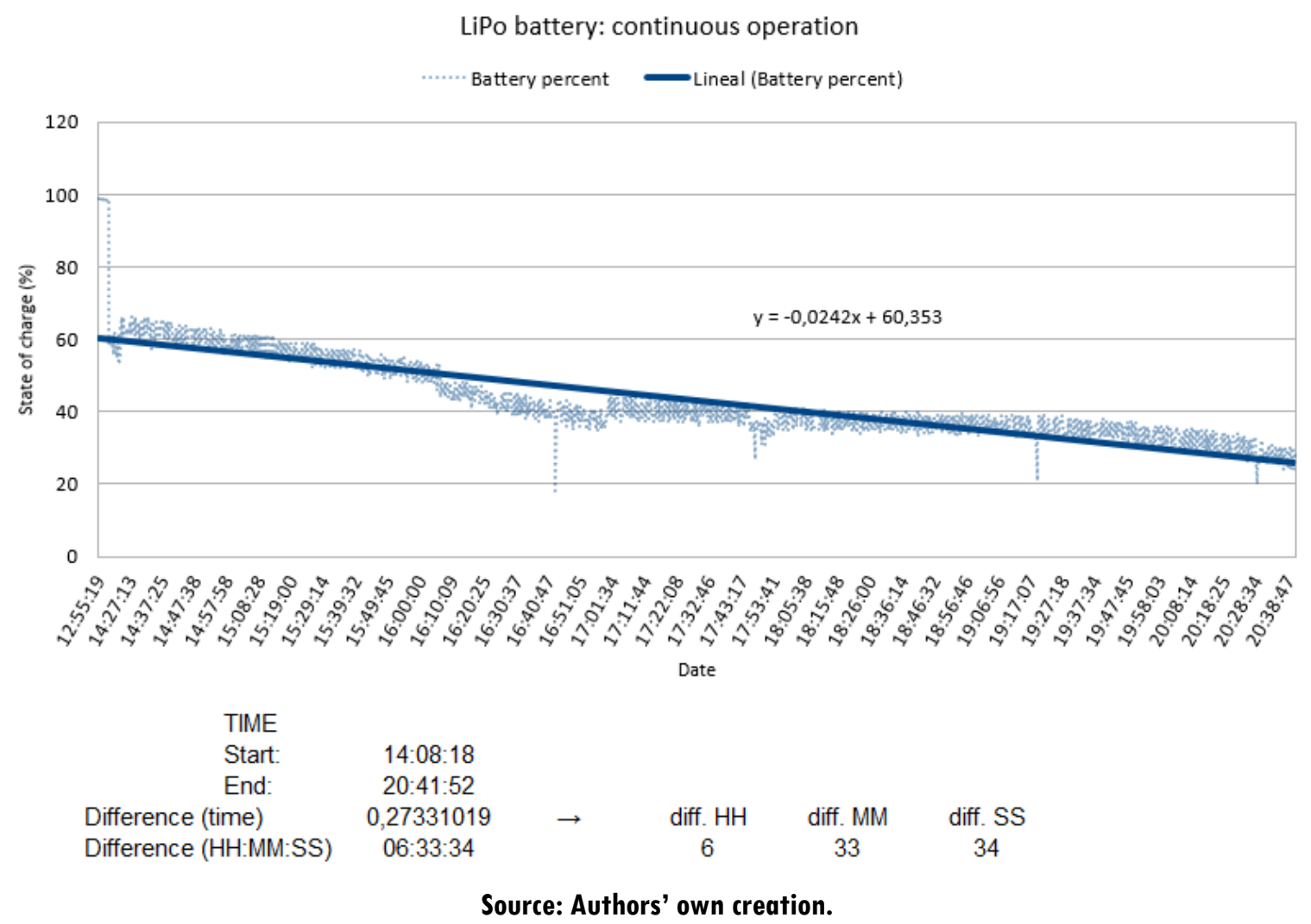

These tests were optimal concerning the transmission of data since the PLA material did not present any interference with the cellular signals or GPS, achieving a stable and constant connection. Having a low number of disconnections, it was not possible to differentiate whether it was due to the encapsulation or if it was related to breakdowns associated with the cellular network. However, it was necessary to change the battery to ensure that the module could work continuously for an 8-hour day, so finally, the capacity of the battery employed was $2000 \mathrm{mAh}$ in the final prototype.

\section{Third test stage}

Once the improvement tests in the programming code were completed, performance tests were carried out considering the possible environments that the module could hold, for which an aluminum-clad wooden box was prepared, and thus, generated installation restrictions, in addition to presenting design parameters for a definitive encapsulation.

For correct representation, the previously performed route was iterated, with multiple variations in the location of the module inside the box; however, these can be summarized in four groups. 


\section{Full aluminum-clad enclosed, horizontal module}

The container was completely covered to simulate an incorrect installation location as if it were a vehicle whose body was entirely made of metal, which provides an unstable connection to the cellular network and a null possibility of obtaining the location of the GPS. Figure 11 shows both the proof of concept of the encapsulation and the result of this, although a data transmission was achieved, many disconnections were recorded.

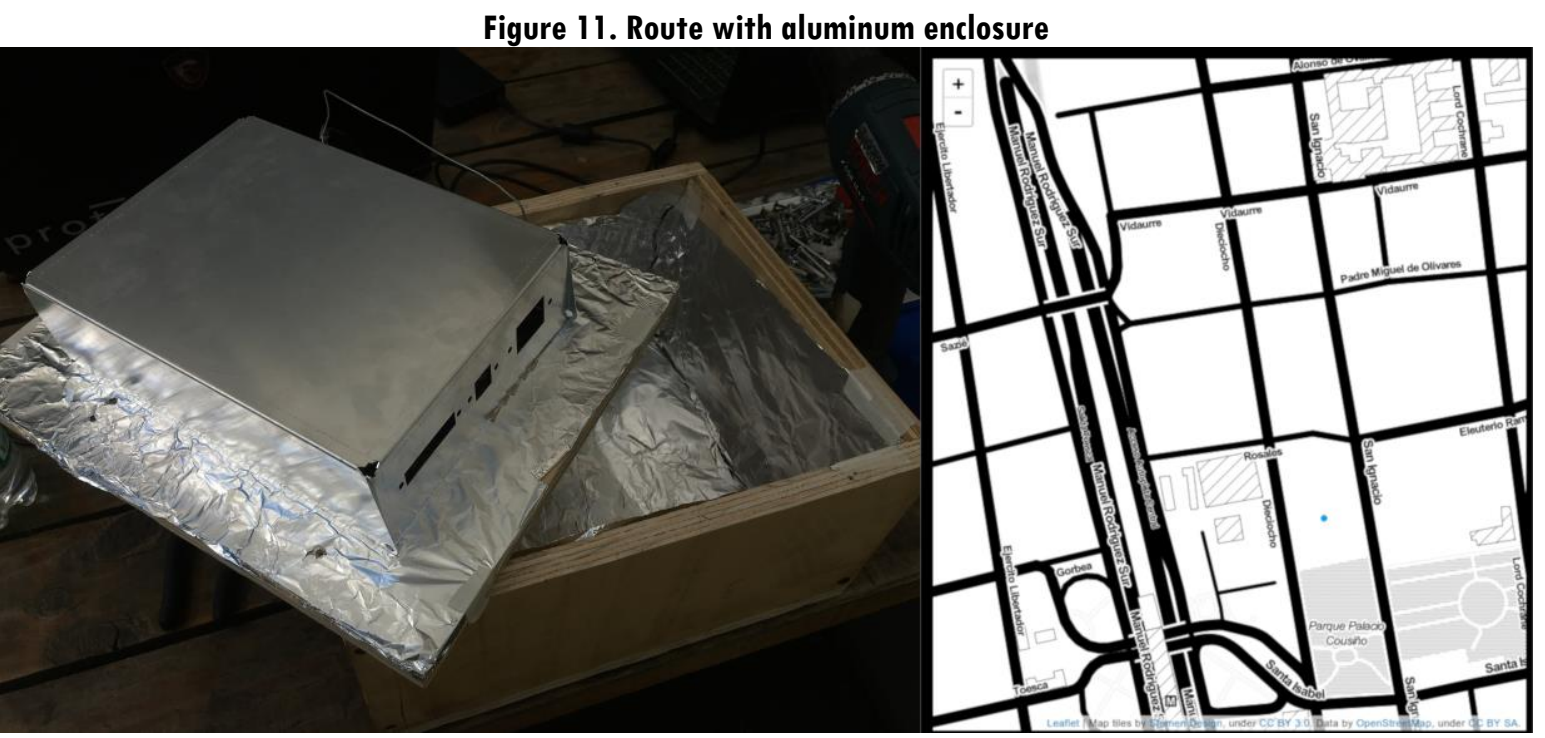

Source: Authors' own creation.

In thermal terms, the device was coupled to the expected requirements but limited communication to the cellular network.

Encapsulated in aluminum, with acrylic cover and horizontal location

In this case, two tests were carried out. First, the module was mounted in the bottom of the box with only a $12 \mathrm{~mm}$ plywood lid, which provided an excellent connection to the cellular network, but the coordinates provided by the GPS tended to be irregular, so it was concluded that there were synchronization problems with satellites. Subsequently, the module was attached to the lid with an aluminum window the size of the area of the device; with this, excellent communication was achieved with the cellular network, and no significant problems were observed with the GPS location information.

In thermal terms, the device was coupled to the expected requirements, improving the previous condition related to the establishment of communication with the cellular network. 


\section{Encapsulation, with coating and lateral location}

The device was tested by placing the module sideways, but leaving the lid free of aluminum; this resulted in a large number of disconnections and location problems, with worse results than those observed in the box without aluminum cover and with the device in the bottom.

\section{An encapsulated prototype, the final module}

Finally, in its last iteration of encapsulation, all the considered observations were taken according to the behavior of the device and its relation with the container materials (see Figure 12). The degree of the position of the prototype with the operation of the connectivity and the relationship that exists between the reduction of obstruction of components with the antennas used for both GPS and GPRS was also considered.

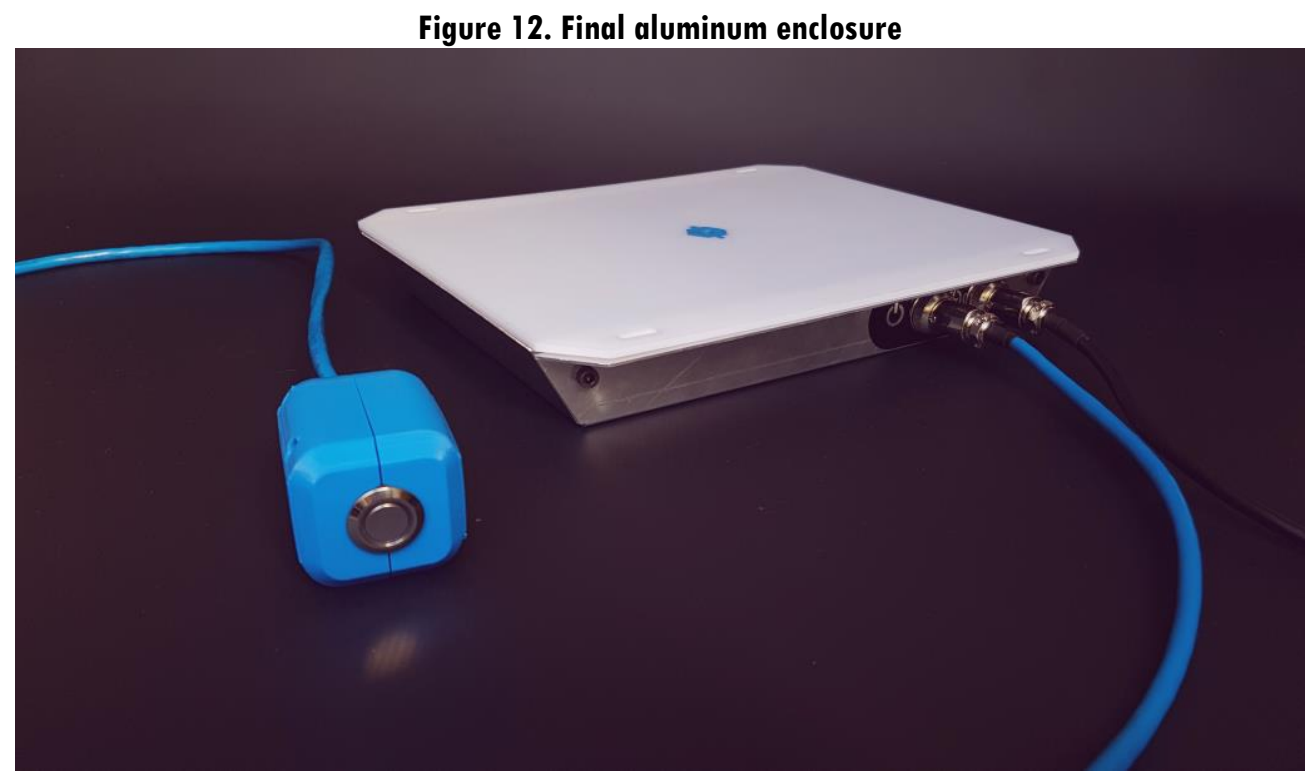

Source: Authors' own creation.

From all those considerations, an encapsulation was prepared that contained the communication module and allowed its installation inside a light-duty vehicle for its function of monitoring and enabling function to observe the environmental parameters of the cargo area, obtaining a record as you can see in Figure 13, where the route marks the trace of the tracking device without erratic readings in terms of connectivity, thermal characteristics, and availability of the transmitted data. 
Figure 13. Route with final aluminum enclosure

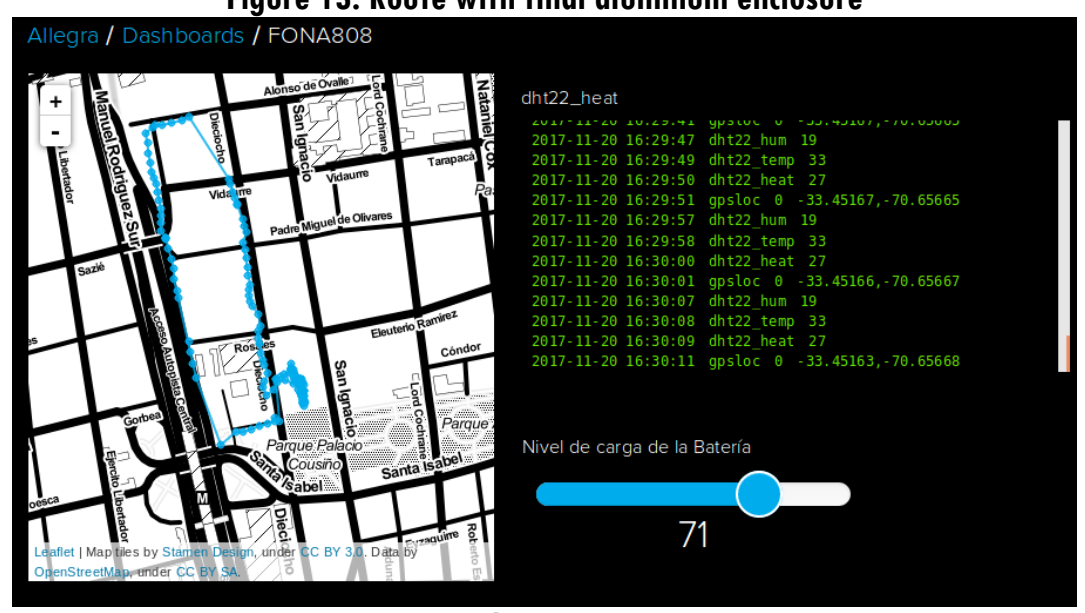

Source: Authors' own creation.

\section{Future works}

The prototype projection regarding the use of services in the cloud, such as Azure, AWS, or IBM Bluemix, was found in the research. The module only achieves data transmission via the GSM network and the implementation of secure ciphers such as SSL and TLS. There are, therefore, two development possibilities. A first option concerns developing libraries for the direct implementation of the Arduino development boards, with which a processing load can be generated. This processing load can surpass the capacities of the microcontroller. A second option is found in the functions of cloud services, which possess a great number of tools for IoT, adding stages that allow the unsafe reception of data, but adding an encryption phase necessary for protecting the information obtained by the prototype.

\section{Conclusions}

The data communication tests and establishments linking to the GPS location system were successful according to the latest encapsulation projection for the electronic device. For the Chilean national context, the use of the GSM network is still valid for the transmission of information, which is an optimal result for the work proposal. The value proposition of making a device that is governed by the proposed restrictions was validated through a continuous improvement methodology applied both in the programming stage and prototype design. The thermal variant was also confirmed in the tests indicated into section "Third stage test", conditioning that the installation of the device must be in the upper part of the transport car. It is in this last point that the considerations for establishing communication are a priority when using the prototype tracking and localization. Finally, integrating the prototype proposed in this work through a cloud service for storage, reporting and analytics allowed rapid commissioning to validate ideas of new services in environments of entrepreneurship or innovation, such as digital manufacturing centers that belong to academic institutions. 


\section{References}

[1] P. Daud, J. A. Simalongo, D. Mahmudin, Y. N. Wijayanto, and P. Putranto, "Design of vehicle tracking system using MCU STM32F103RET6 and SIM908,” Internetworking Indones. J., vol. 9, no. 2, pp. 3-7, 2017.

[2] V. Bosetti and T. Longden, "Light duty vehicle transportation and global climate policy: The importance of electric drive vehicles," Energy Policy, vol. 58, pp. 209-219, Jul. 2013.

[3] S. Liawatimena and J. Linggarjati, "Vehicle tracker with a GPS and accelerometer sensor system in Jakarta,” Internetworking Indones. J., vol. 9, no. 2, pp. 9-15, 2017.

[4] Ministerio de Salud de Chile, "Decreto 977: aprueba reglamento sanitario de los alimentos." Chile: Biblioteca del Congreso Nacional de Chile, 2019, p. 183.

[5] Seremi Salud, “Cadena de Frío: Programa Nacional de Inmunizaciones," Seremi Salud, 2015. [Online]. Available: http://www.cmvm.cl/content/salud/biblioteca/clases2018/Cadena de Frio.pdf. [Accessed: 24-Jun-2018].

[6] T. Godavari and J. Umadevi, "Cloud computing based real-time vehicle tracking and speed monitoring system,” Int. J. Control Theory Appl., vol. 9, no. 4, pp. 1823-1830, 2016.

[7] M. S. Rosdi and N. Ahmad, "Smart handbag system with location tracking," ARPN J. Eng. Appl. Sci., vol. 11, no. 18, pp. 10840-10844, 2016.

[8] P. Yi, L. Huafu, F. Lu, Z. Zhuxian, H. Feijiang, and C. Chenglin, "A GPS/GSM Based Vehicle Monitoring and Anti-Theft System,” Int. J. Smart Home, vol. 10, no. 7, pp. 115-124, Jul. 2016.

[9] R. Fernández-Garcia and I. Gil, "An alternative wearable tracking system based on a low-power wide-area network," Sensors, vol. 17, no. 3, p. 592, Mar. 2017.

[10] SIMCOM.EE, "SIM808 Specifications," GSM / GPRS + GNSS Modules, 2015. [Online]. Available: https://simcom.ee/documents/SIM808/SIM808 SPEC_V1507.pdf. [Accessed: 20-May2018].

[11] G. Association, "The mobile economy 2019," 2019.

[12] H. Pranjoto, L. Agustine, and M. Mereditha, "OBD-II-based vehicle management over GPRS wireless network for fleet monitoring and fleet maintenance management," J. Telecommun. Electron. Comput. Eng., vol. 10, no. 2-3, pp. 15-18, 2018.

[13] M. K. Balga and F. Başçiftçi, "REST API based image processing interactive person tracking application," in 2nd International Conference on Computer Science and Engineering, UBMK 2017, 2017, pp. 22-27.

[14] N. Velasquez, C. Medina, D. Castro, J. C. Acosta, and D. Mendez, "Design and development of an IoT system prototype for outdoor tracking," in Proc. of the International Conference on Future Networks and Distributed Systems - ICFNDS '17, 2017, pp. 1-6. 
[15] Z. Rezvani, J. Jansson, and J. Bodin, “Advances in consumer electric vehicle adoption research: A review and research agenda,” Transp. Res. Part D Transp. Environ., vol. 34, pp. 122-136, 2015.

[16] M. Anil Kumar, M. Venkata Suman, Y. Misra, and M. Geetha Pratyusha, "Intelligent vehicle black box using IoT," Int. J. Eng. Technol., vol. 7, no. 2, pp. 215-218, 2018.

[17] C. C. Chan, "The state of the art of electric and hybrid vehicles," Proc. IEEE, vol. 90, no. 2, pp. 247-275, 2002.

[18] C. C. Chan, "The state of the art of electric, hybrid, and fuel cell vehicles," Proc. IEEE, vol. 95, no. 4, pp. 704-718, Apr. 2007.

[19] T. Hutchins, "EIA Conference: Light -duty vehicle energy demand, demographics, and travel behavior," in EIA Conference, 2014.

[20] L. Fried, “Overview | Adafruit FONA 808 Cellular + GPS Shield for Arduino,"” ADAFRUIT.COM, 2015. [Online]. Available: https://learn.adafruit.com/adafruit-fona-808cellular-plus-gps-shield-for-arduino. [Accessed: 02-Feb-2018].

[21] O. A. Allah, M. Abdalla, S. Abdalla, A. Babiker, and A. A. Allah, "Universal data logger system for environmental monitoring applications," Indones. J. Electr. Eng. Informatics, vol. 5, no. 2, pp. $131-136,2017$.

[22] R. R. Yeddula and P. Vetrivelan, "Internet of vehicles-based real-time vehicle tracking," Asian J. Pharm. Clin. Res., vol. 10, pp. 57-60, 2017.

[23] T. Yokotani and Y. Sasaki, "Comparison with HTTP and MQTT on required network resources for IoT," in 2016 International Conference on Control, Electronics, Renewable Energy and Communications (ICCEREC). https://doi.org/10.1109/ICCEREC.2016.7814989 